\title{
Knowledge and Understanding of Type 1 Diabetes and Its Management among Saudi Children and Adolescents
}

\author{
Latifa Aldossary $^{1}$, Sherrill Snelgrove ${ }^{2}$ \\ ${ }^{1}$ Imam Abdularhman bin Faisal University, Dammam, Saudi Arabia \\ ${ }^{2}$ College of Human and Health Sciences, Swansea University, Swansea, United Kingdom \\ Email: laldossari@iau.edu.sa,s.r.snelgrove@swansea.ac.uk
}

How to cite this paper: Aldossary, L. and Snelgrove, S. (2020) Knowledge and Understanding of Type 1 Diabetes and Its Management among Saudi Children and Adolescents. Journal of Diabetes Mellitus, 10, 1-15.

https://doi.org/10.4236/jdm.2020.101001

Received: December 3, 2019

Accepted: January 28, 2020

Published: January 31, 2020

Copyright $\odot 2020$ by author(s) and Scientific Research Publishing Inc. This work is licensed under the Creative Commons Attribution International License (CC BY 4.0).

http://creativecommons.org/licenses/by/4.0/

\begin{abstract}
Introduction: globally there is an increase of Type 1 diabetes among children. Poorly controlled Type1 diabetes is associated with the complication. Knowledge is a crucial factor in maintaining patient glycaemic control and adherence to treatment. Purpose: to explore knowledge and understanding of Type 1 diabetes and its management among children in Saudi Arabia. Methods: 32 semi structured interviews conducted with children and their parents. It was audio recorded and transcribed. The qualitative data was thematically analysed. NVivo 11 software was used to organise transcript. Results: Children and young people know the aggravating factors and the theoretical aspects of T1DM management but fail to adhere to the treatment regimen practically. Children and adolescents preferred school food; however, parents complained about the quality of school food. Conclusion: The study findings indicate a requirement to employ innovative and engaging ways of communicating with children and adolescents concerning the promotion of a positive lifestyle and healthy practices. There is a need for cooperation between schools and health ministries to increase awareness about T1DM management and complications.
\end{abstract}

\section{Keywords}

Type 1 Diabetes, Knowledge, Understanding, Management, Children

\section{Introduction}

Type 1 diabetes is an immunodeficiency disease where the body's immune system attacks the insulin-producing beta cell in the islet of the pancreas gland [1] 
More than 180 million people worldwide have diabetes [1]. Unfortunately, it is very likely that this number will be more than double by 2030 [2]. The incidence of Type 1 diabetes has been increasing all over the world, including Saudi Arabia [3]. The prevalence of Type 1 diabetes among Saudi children was estimated by 109.5 per 100,000 [3].

Knowledge is a crucial factor in maintaining patient glycaemic control and adherence to treatment. However, poor glycaemic control, insufficient treatment adherence and severe psychological adjustment to disease could result from inaccurate or lack of knowledge about diabetes risk [4]. In Saudi's literature, only two quantitative studies performed to evaluate children and adolescent's knowledge on their Type 1 diabetes. Both studies found participants had lack of knowledge and that has a potential impact on their ultimate control and health management efforts [5] [6]. However, the reasons for that poor knowledge among children were not understood. Therefore, this study aims to explore knowledge and understanding of Type 1 diabetes and its management. The qualitative method used in this study allows children and adolescents to express their views about their Type 1 diabetes.

\section{Study Methods and Materials}

The study was conducted in two children diabetic clinic in Eastern region hospitals in Saudi Arabia between February and May 2017. The sampling technique was purposive sampling. The participants' total number was 32 from both hospitals. The sample of the study is divided into two main categories: 1) children and adolescents who have been diagnosed with T1DM 2) people who have direct contact with or responsibility for those children, that is, their parents and/or guardians.

The inclusion criteria were children and adolescents aged 5 to 16 years. The reasons to choose these age groups are five-year-old children will be able to speak clearly, and independence is increasing. The paediatric participants had all been diagnosed with T1DM. Furthermore, the parents of the children were also included in the study. The health team currently giving health promotion advice and guidance in the clinics were also selected.

Informed consent and assents were obtained from the participants before conducting semi structured interviews. All children and parent interviews were in private place. Children were separately interviewed unless the child insists on his/her parent present. The questions in the interview were open-ended questions. The tool was sent to an expert group in Saudi Arabia to confirm that questions fit the research aim, and to be sure that language of the interview was adjusted according to the participants. Average interview time was 45 minutes.

All the children were given anonymous names. Interviews were audio recorded and transcribed. The two diabetic clinics were given abbreviation as HA and HB. NVivo 11 software was used to organise transcript. All the data were thematically analysed. 
Ethical approval was obtained from Ministry of Health Ethics Committee at Saudi Arabia and from the College of Human and Health Sciences Ethics Committee at Swansea University.

\section{Results}

The 32 semi structured interviews were conducted in two large hospitals. In both hospitals, the mothers' number $(n=14)$ was more than fathers $(n=2)$. Majority of parents were married. The median age of children and young people was 12.5 years in Hospital A and 12 years in Hospital B. Participants demographic data summarized in Table 1.

Analysis of the data revealed three main themes related knowledge of Type 1 diabetes and its managements. 1) Children and parent understanding of Type 1 diabetes, 2) children and adolescents understanding of Type 1 diabetes management, 3) Managing diabetes within Saudi Arabian culture. A summary table for the themes (Table 2).

Main Theme 1: Children and parent understanding of Type 1 diabetes.

All the children and adolescent participants spoke about their understanding of type 1 diabetes, in response to the question "what you think diabetes type 1

Table 1. Parent and CYP demographic data.

\begin{tabular}{|c|c|c|c|c|}
\hline \multirow[b]{2}{*}{ Characteristics } & \multicolumn{2}{|c|}{ Parent $(n=16)$} & \multicolumn{2}{|c|}{ CYP $(n=16)$} \\
\hline & $\begin{array}{c}\text { Hospital A } \\
\text { n (\%) }\end{array}$ & $\begin{array}{c}\text { Hospital B } \\
\text { n (\%) }\end{array}$ & $\begin{array}{c}\text { Hospital A } \\
\text { n (\%) }\end{array}$ & $\begin{array}{c}\text { Hospital B } \\
\text { n (\%) }\end{array}$ \\
\hline \multicolumn{5}{|l|}{ Gender } \\
\hline Female & $7(43.75)$ & $7(43.75)$ & $6(37.50)$ & $4(25.00)$ \\
\hline Male & $1(06.25)$ & $1(06.25)$ & $2(12.50)$ & $4(25.00)$ \\
\hline \multicolumn{5}{|l|}{ Age in year } \\
\hline Median & 40 & 40 & 12.5 & 12 \\
\hline (Range) & $(37-45)$ & $(32-49)$ & $(8-14)$ & $(7-13)$ \\
\hline \multicolumn{5}{|l|}{ Education level } \\
\hline Primary school & $0(00.00)$ & $0(00.00)$ & $4(25.00)$ & $5(31.25)$ \\
\hline Intermediate school & $0(00.00)$ & $1(06.25)$ & $4(25.00)$ & $3(18.75)$ \\
\hline Secondary school & $3(18.75)$ & $4(25.00)$ & $0(00.00)$ & $0(00.00)$ \\
\hline Bachelor's degree \& above & $5(31.25)$ & $3(18.75)$ & $0(00.00)$ & $0(00.00)$ \\
\hline \multicolumn{5}{|l|}{ Marital status } \\
\hline Married & $7(43.75)$ & $7(43.75)$ & & \\
\hline Divorced & $1(06.25)$ & $0(00.00)$ & - & 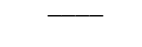 \\
\hline Widow & $0(00.00)$ & $1(06.25)$ & & \\
\hline \multicolumn{5}{|c|}{ Children age at diagnosis in years } \\
\hline$<2$ & & & $1(06.25)$ & $1(06.25)$ \\
\hline $2-5$ & 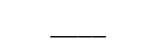 & $\underline{-}$ & $1(06.25)$ & $1(06.25)$ \\
\hline $6-9$ & & & $2(12.50)$ & $5(31.25)$ \\
\hline $10-12$ & & & $4(25.00)$ & $1(06.25)$ \\
\hline \multicolumn{5}{|l|}{ Family history with T1DM } \\
\hline Yes & $5(31.25)$ & $1(06.25)$ & - & - \\
\hline No & $3(18.75)$ & $7(43.75)$ & & \\
\hline
\end{tabular}

${ }^{\star} \mathrm{CYP}$ children and young people, Hospital A is educational hospital, Hospital B is governmental hospital. 
Table 2. Themes summary for the study.

\begin{tabular}{|c|c|c|}
\hline Main Theme & Categories & Main Result \\
\hline \multirow[b]{2}{*}{$\begin{array}{l}\text { Children and parent } \\
\text { understanding of } \\
\text { Type } 1 \text { diabetes }\end{array}$} & $\begin{array}{l}\text { Children and young } \\
\text { people's conceptual } \\
\text { understanding of } \\
\text { T1DM }\end{array}$ & $\begin{array}{l}\text { Six children out of } 16 \text {, aged } 11 \text { and } \\
\text { above were able to explain the cause of } \\
\text { diabetes and mechanism of disease. }\end{array}$ \\
\hline & $\begin{array}{l}\text { Parental knowledge } \\
\text { about T1DM }\end{array}$ & $\begin{array}{l}\text { Seven parents out of } 16 \text {, articulated } \\
\text { that while they do have knowledge of } \\
\text { diabetes, they are unable to control } \\
\text { diabetes or control their children's } \\
\text { behaviour to maintain appropriate } \\
\text { blood sugar levels. } \\
\text { Other six parents expressed that their } \\
\text { knowledge is inadequate. }\end{array}$ \\
\hline \multirow{3}{*}{$\begin{array}{l}\text { Children and young } \\
\text { people understanding of } \\
\text { the management of Type } 1 \\
\text { diabetes }\end{array}$} & $\begin{array}{l}\text { I use s glucometer to } \\
\text { monitor my blood } \\
\text { sugar }\end{array}$ & $\begin{array}{l}\text { The majority of children were } \\
\text { understood the role of } \\
\text { glucometer. However, the } \\
\text { frequency of using glucometer was } \\
\text { varied and children have different } \\
\text { answers. }\end{array}$ \\
\hline & $\begin{array}{l}\text { I take insulin using the } \\
\text { orange and blue and } \\
\text { grey pen }\end{array}$ & $\begin{array}{l}\text { Nine out of } 16 \text { children and adolescents } \\
\text { referred to the insulin regulator in } \\
\text { terms of the colour of the insulin pen } \\
\text { such as orange or a blue and grey pen. }\end{array}$ \\
\hline & $\begin{array}{l}\text { Reading blood sugar } \\
\text { levels, feeling } \\
\text { symptoms, and } \\
\text { managing } \\
\text { hypoglycaemia }\end{array}$ & $\begin{array}{l}\text { Most children in this study } 14 \text { out of } 16 \\
\text { were able to identify hypoglycaemia } \\
\text { symptoms. However, three adolescents } \\
\text { consume a significant amount of } \\
\text { carbohydrate when managing low } \\
\text { blood glucose. }\end{array}$ \\
\hline \multirow{3}{*}{$\begin{array}{l}\text { Main theme } 3 \text { : } \\
\text { Managing diabetes within } \\
\text { Saudi Arabian culture }\end{array}$} & $\begin{array}{l}\text { Children's food } \\
\text { preferences }\end{array}$ & $\begin{array}{l}\text { Eight out of } 16 \text { children and } \\
\text { adolescents were preferred western } \\
\text { diet such as burger, and pizza. } \\
\text { Only two adolescents were use } \\
\text { Carb counting technique in } \\
\text { preparing their meals. }\end{array}$ \\
\hline & $\begin{array}{l}\text { Sedentary life versus } \\
\text { being active }\end{array}$ & $\begin{array}{l}13 \text { out of } 16 \text { of the children and } \\
\text { adolescents were active and did } \\
\text { exercises every day. } \\
\text { Few }(n=3) \text { teens girls were prefer } \\
\text { sitting at home because of hot weather. }\end{array}$ \\
\hline & $\begin{array}{l}\text { School food } \\
\text { environment }\end{array}$ & $\begin{array}{l}(\mathrm{n}=13) \text { of children and adolescents } \\
\text { prefer school food because it is } \\
\text { attractive for them. }\end{array}$ \\
\hline
\end{tabular}

means?".

1) Children and young people's conceptual understanding of T1DM

Six children aged 11 years and above were able to identify the cause of the disease originating from the pancreas as an organ essential for secreting insulin to regulate blood glucose levels: 
"It means that my pancreas does not produce or secrete insulin, and I have to take insulin by injection" (Zaid 14 yrs, HA).

"Diabetes ...aah... when my pancreas does not produce insulin, and my blood sugar increased" (Laila, 11yrs, HB).

However, younger children in this study were not able to identify the organ or mechanism of action. These children expressed their understanding in a simple word, made reference to diet and insulin control or described their symptoms of diabetes:

"I do not know. Mm, diabetes is no sugar and injection" (Soha, $7 \mathrm{yrs}, \mathrm{HB}$ ).

"Diabetes means no sweet food and insulin injection" (Nouf, 8 yrs, HA).

Moreover, two older children replied that the disease could occur to anyone or referred to sugar as the external agent responsible for their diabetes and they gave vague explaination:

"Diabetes is a disease which could occur to anyone; I do not know why" (Rana, 11 yrs, HA).

"Ummm (laugh) it means I do not know, but I think it results from eating sugar too much" (Leen, 12 yrs, HA).

The other children were explained symptoms or managment for Type 1 diabetes:

"Ahhhhh, diabetes is to maintain and regulate my diet to avoid an increase in sugar" (Omar, 13 yrs, HB).

"Oh, diabetes is when I cannot see, and I went to the toilet too many times" (Saif, 11 yrs, HB).

2) Parental knowledge about T1DM

The skills and capacity of parents to manage and handle the self-care activities of their children were linked to their understanding and awareness of Type 1 diabetes [7]. Therefore, parent participants were asked to describe their knowledge of T1DM, three out of 16 suggested they know and understand T1DM and how to care for their children:

"Oh, yes, I like reading so much as I graduated from Math College, but my dream was Medicine College, but I could not enter. Therefore, I see myself that I am knowledgeable about treatment, and control of diabetes. Now my daughter HAlc become 6.8 before it was started with, uh, ten and with good control it starts to decrease" (Leen's mother, HA).

While six expressed that, their knowledge is weak or inadequate:

"My knowledge is little; I only know how to inject and what to do with low blood sugar" (Saeed's mother, HB).

On the other hand, the majority of parents, in the present study, articulated that while they do have knowledge of diabetes, they are unable to control diabe- 
tes or control their children's behaviour to maintain appropriate blood sugar levels:

"I know what diabetes and treatment are and when it is low or high. I think it is good, but I do not know how to control it $\mathrm{mmm}$ difficult" (Raja's mother, $\mathrm{HB}$ ).

"I think, it is good. I know what diabetes is, and the treatment is insulin and how to act during a low blood sugar level. On the other hand, I cannot control my child's diet and activity." "My daughter becomes stubborn, sometimes refuse to eat what I prepared for her, and at school, she eats with her friend's junk food." (Nouf's mother, HA)

In this study, two fathers from both hospitals agreed to participate. One of them claimed he had a good knowledge of diabetes and was involved in the management of his child. The other father gives reasons for his inadequate information about diabetes:

"Ah, it is poor because my wife has the most responsibility to take care of him. I only take him to his clinic appointment if she was busy" (Zaid's father, HA).

While the second father mentioned that:

"My knowledge is good... and I get this knowledge from visiting the health team clinics. My son achieved better glycaemic control" (Majed's father, $\mathrm{HB})$.

Main Theme 2: Children and young people understanding of the management of Type1 diabetes.

Children and adolescents expressed their understanding of Type 1 diabetes management. They use the following expression.

1) I use s glucometer to monitor my blood sugar

Around 12 out of $16(80 \%)$ of children and adolescents who responded to the question "can you explain to me what is at Glucose meter (finger prick test)?" understood the primary role of the glucose meter (Finger Prick Test) in diabetes which is to measure the amount of glucose in the blood:

"I use this to measure blood sugar before I take insulin injection" (Safa, 12 yrs, HA).

However, only around one-third (five out of 16) of older children mention the frequency of doing a blood glucose test per day and their answers varied from 2 or 3 to 4 or 6 times and more, or before and after the meal, typical responses were:

"I use this to measure blood sugar, usually before injection time. Mm, I do it sometimes three times or two times" (Noha, 13 yrs, HA).

"I do finger prick test from 3 - 4 times per day to see blood sugar level, and then I prepare my insulin dose" (Leen, 12 yrs, HA). 
From all the participants in my study only two children reported pain from this procedure:

"My mum does it for me. I do not like it mmm caused pain" (Soha, 7 yrs, $\mathrm{HB})$.

"This is the device I use it to measure my sugar level, I used it around three times per day and sometimes only two because it causes pain" (Raja, 12 yrs, $\mathrm{HB})$.

2) I take insulin using the orange and blue and grey pen

Most participants from both hospitals had taken insulin via an insulin pen. Nine out of 16 children and adolescents referred to the insulin regulator in terms of the colour of the insulin pen such as orange or a blue and grey pen.

"Uhh, I do not know the name, but it is the pen one which is orange for the eating time, and other is the regulator pen I take it at 8:00 pm" (Leen, 12 yrs, HA).

"(mmm, looking at her mum), I take orange and grey pen" (Soha, 7 yrs, $\mathrm{HB})$.

In contrast, four older children aged 13 years and above were able to memorise Lantus as an insulin regulator.

"I took the orange pen and the grey pen... I know only Lantus (grey colour) easy to pronounce" (Noha, $13 \mathrm{yrs}, \mathrm{HA}$ ).

Two of the participants mentioned that they use an insulin pump and expressed feelings of freedom from the daily injection while another child said that he deserved the insulin pump because of his adherence and control to T1DM, in this clinic insulin pumps are allocated according to children adherence to treatment, their parent educational level and stability of social life:

"I have insulin pump but before I was using the pen... after insulin pump, I feel free" (Eman, 14 yrs, HA).

"I have an insulin pump, I was using a pen, and for three years, I have a pump... I deserve an insulin pump because of my ability to control and manage diabetes" (Majed, 12 yrs, HB).

3) Reading blood sugar levels, feeling symptoms, and managing hypoglycaemia

Children and adolescents from both hospitals reported low and high blood glucose in different ranges such as:

"Reading less than 80 is low blood sugar and if reading more than 300 is high" (Noha, 13 yrs, HA).

"When my sugar $140 \mathrm{mg} / \mathrm{dl}$ is good, $300 \mathrm{mg} / \mathrm{dl}$ is high, and $50 \mathrm{mg} / \mathrm{dl}$ is low blood sugar” (Saif, 11 yrs, HB).

Around 14 out of 16 children in the study were able to express the symptoms 
they feel either when they have low blood glucose or high blood glucose. These symptoms include a headache, dizziness, tired or thirsty.

"Uh, I feel chills and dizziness if low blood sugar and I feel thirsty when it is high" (Noha, 13 yrs, HA).

Another child answered:

"I feel dizzy when my blood sugar is low, and I feel [pause 2 seconds] my tongue is heavy, I cannot talk when my blood sugar is high" (Rana, 11 yrs, HA).

Most of the children and adolescents in the study indicated that they manage low blood sugar by eating sweet foods, like chocolate, honey, juice. However, they did not clarify the amount of consuming high carbohydrate food. For example:

"I will take something to raise it up, for example, I drink juice, or eat sweets" (Noha, 13 yrs, HA).

"I eat chocolate" (Nouf, 8 yrs, HA).

However, three adolescents consume a significant amount of carbohydrate at the same time when managing low blood glucose as reported by:

"My mum gives me honey or juice and bread" (Ali, 13 yrs, HA).

"I take juice then check my blood sugar then I take dates every hour" (Rula, 13 yrs, $\mathrm{HB})$.

Only two adolescents who followed the 15-minute rule to manage their low blood glucose:

"After I am sure that it is low, I took a spoon of honey, or I drink juice, after 15 minutes I checked again if my blood sugar starts to elevate, I eat a small snack such as a half bread with juice" (Eman, $14 \mathrm{yrs}, \mathrm{HA}$ ).

\section{Main Theme 3: Managing diabetes within Saudi Arabian culture.}

Children and adolescents spoke about the way of managing their diabetes within Saudi culture.

1) Children's food preferences

T1DM dietary management is essential, as food needs should be corresponding with insulin needs to avoid high sugar levels or lower than average. From the interviews around half of the participants were following a healthy diet either by doing a carbohydrate count or by preparing small healthy portions, here are some of their responses:

"My plate fill of all food components such as little of rice, a small part of chicken or fish, fruit and vegetable except for tomato I do not like it, and I always drink a lot of water, around ten bottles of these (pointing at water $330 \mathrm{ml}$ bottle she holds)" (Rana, 11 yrs, HA).

It was surprising that only two young people Majed and Eman were able to 
use the Carb count technique in preparing their food.

"I eat what my mum cooks and I help her in counting carb" (Eman, 14 yrs, HA).

"...after I count the carb, I enter on my pump the amount" (Majed, 12 yrs, $\mathrm{HB})$.

On the contrary eight out of 16 CYP participants expressed their preference for a western diet such as burger, chips and pizza:

"I eat for example plate of rice or pasta with chicken, but no vegetables or fruit I do not like them, I do not drink water. I like juice more. I prefer fast food; burger and chips" (Safa, 12 yrs, HA).

"I like to eat cereals, banana and to buy from the supermarket juice, chips, and soft drink" (Saif, 11 yrs, HB).

Further, some of the adolescents had poor eating habits, and they expressed that they do not like to eat fruit or vegetables, or they drink energy or soft drinks:

"I eat for example plate of rice or pasta with chicken, but no vegetables or fruit I do not like them. I prefer energy or soft drinks." (Safa, 12 yrs, HA)

Parents responded to questions about how they manage their family meal. Six out of 16 of the parents adopt the health team strategies of managing the food that their children with diabetes eat and find a way to manage their family's meal and their lifestyle become organised:

"After her diagnosis, I started to change gradually and organise our meal and type of food... For example, before the diagnosis, we were ordering fast food and drinking soft drinks, and our fridge contains chocolate and other sweets. However, now is different our fridge filled with fruit and vegetables. Also, my cooking style changed. So, I make sure that our meal contains all food elements and I advise my children to drink much water." (Eman's mother, HA)

This in contrast with an eight years old child who indicated that the diet changed for her only:

"My mum put for me a small amount in plate different than my brothers. Yes, I eat a plate of salad every day. I drink much water, and I do not know how many" (Nouf, 8 yrs, HA).

Also, a mother of an adolescent spoke of changing the diet for her child after the appearance of a liver problem:

"Yes, ahhhI started to change it and decrease everything, mmm I decrease using oil, andI avoid it, because of his last test diabetes affect his liver, also I decrease the amount of food for him. For the sugar, I remove it from his diet, but for us we still adding sugar to our food" (Omar's mother, HB). 
Further, eight out of 16 of the parents indicated that their family meal was not changed because of their children food preferences or they did change only the type of cooking oil:

"Well, I did not change my cooking. I tried at the beginning of the diagnosis, but my daughter did not accept it, and I do not want them to feel that they are different from us. In the lunchtime, for example, you will find Coke, Pepsi, and chips. That what they are wanted to eat, or they will order by phone fast food" (Noha's mother, HA).

2) Sedentary life versus being active

Around 13 out of 16 of the children and adolescents were active and did exercises every day. They mention different type of activities such as walking, riding the bicycle, or play football with friends:

"I have a bicycle, I played near my house with my big brother for 30 minutes, or sometimes we go for a walk" (Rana, 11 yrs, HA).

"I like playing outside the home; every day I take my bicycle or play football with my friends" (Saif, 11 yrs, HB).

Three female adolescents preferred playing on their electronic devices or watching TV rather than walk or exercise in hot weather said as the following:

“...I prefer setting watching my iPad" (Noha, 13 yrs, HA).

"I do not do exercise; I prefer to set on I pad rather than walk in hot weather" (Raja, 12 yrs, HB).

"I do not exercise; it is hot outside" (Laila, 11 yrs, HB).

One teenager girl in the sample, wished to perform physical activity, but due to a social and cultural circumstance, she could not practice it:

"I would like to do exercise, [silent for 5 seconds], I cannot do it because my grandfather not allows me to go outside for a walk... No, he will not allow for my mum and me to go alone for a walk alone without a man attending with us" (Safa, 12 yrs, HA).

3) School food environment

When I asked children and adolescent about their eating habits during school time, most of the participants (13 out of 16) said they would choose school food because it is more attractive to them as:

"In school, I buy my food from the school. Everything is there; you will find a different kind of sandwiches, pastries, chocolate, candies, juices, and soft drinks. It looks like a small restaurant" (Saif, 11 yrs, HB).

Moreover, another comment "I prefer [food] from school. There is a variety of different sandwiches and attractive chocolate and drinks" (Zaid 14 yrs, HA).

On the other hand, only three participants who prefer home prepared food 
because it is healthier than school food:

"Because they only provide food such as chocolate, doughnuts, pastries, chips and juices and in the summer, you would find ice cream... so I prefer my mother's healthy snack" (Majed, 12 yrs, HB).

A parent raised the need for school cooperation and increased awareness in the community and schools about diabetes:

"Yes, I hope if there is a community and school awareness about diabetes type 1 because most of our school provide only junk food that attracts children. Also, I wish if there is a way to control the quality of food that is available in the supermarkets and restaurants" (Majed's father, HB).

\section{Discussion}

The adolescnets offer a typical and straightforward account of their diabetes. They describe how the pancreas malfunction causes diabetes. They name the correct organ and make an association between insufficient insulin and a sugar level increase in the blood and they have to take insulin via injection to compensate for its deficiency. It was proposed that children aged 11 - 16 years give explanations of illness in terms of malfunction of the offending organ [8]. Moreover, people 12 years old and above develop hypothetical, conceptual and abstract thinking which was evident in Zaid's understanding of organ and disease [9]. However, Laila, the 11 years old was also able to understand the organ function which indicates that Piaget might underestimate children cognitive ability. For the youngest children, Soha and Nouf, the meaning of living with diabetes was mainly about avoiding high carbohydrate food and daily insulin treatment.

In contrast to the other older children who understand nature of Type 1 diabetes. Some older children provide vague explanations and attributions for causation are tentatively given as external to themselves. While this is in concordance with Bibace and Walsh's ideas, there is also less understanding about the causes of diabetes than the previous children (Saif and Rana) of a similar age [9]. Some adolescents appear to distance themselves from causality. Rana in particular, suggests it could happen to anyone. She positions herself as non-culpable while Leen thought that the cause of the disease was by consuming a high amount of carbohydrate, although she doesn't directly refer to her own sugar consumption. This may be a useful coping strategy. Studies show that self-blame is associated with disease maladjustment and correlated with distress and depression. A study suggested that adolescents with poor adherence to diabetes management used negative coping strategies such as self-blame or blaming others [10]. It is important to assess CYP's knowledge of their chronic disease because it is claimed that children with chronic illness should have some knowledge that helps them to take some responsibility, control their own health, and lead to better outcomes [11]. 
A few parents claim good knowledge about diabetes. On the other hand, almost third of parent participants who have the necessary basic information for daily management of their children's diabetes including glucose monitoring and insulin injections. However, they suggest that they have very little knowledge other than that needed to manage daily. Several studies show that parental level of education is linked to their children achieving better glycaemic control [12] [13]. Despite the basic theoretical knowledge that these two mothers have, they face difficulties in controlling T1DM or controlling their children behaviour. Some mothers have a good knowledge of T1DM, and they are highly educated. However, they are not able to control glucose levels of their children. A recent study demonstrated that there was a high level of theoretical knowledge for the parents of children and young people with T1DM; however, there was inadequate metabolic control [14]. The findings in the present study appear to support this suggesting poor metabolic control depends on other factors and not only parents' knowledge and further investigation is required. A father expressed that the primary responsibility is with the mother although he will take the child for appointments whenever his wife is busy. In terms of health care of his child, he is the secondary carer, and his wife is the primary carer who has the most responsibility. A recent longitudinal study in southwest England of 14,701 children and their families found that children who have increased father involvement in the care results in positive emotional impact and less behavioural problems of children [15].

Factors related to diet and exercise are the leading causes of poor adherence to the management of Type1 diabetes. Most children and adolescents in the present study who were interviewed adopted a fast-food diet, which is not wholly compatible with diabetic diet requirements. However, some of the families with diabetic children were willing to help their children by changing to healthier diets and organising meals according to the health team's advice. However, some faced resistance to change from their children. Some of the adolescents were noncompliant with a healthy diet. This was usually due to peer pressure or unhealthy school food. Unfortunately, there is no policy to control school food quality in Saudi Arabia and most children and adolescents preferred school food to the healthy meals that their mothers prepared at home. These findings are consistent with previous research that claimed that the main reason why children and adolescents find it difficult to adhere to healthy diabetic-friendly diets is that they often succumb to social pressures, which limit their active involvement in the management of the chronic condition [16]. Children and young people are more concerned with a favourable lifestyle consistent with their peers and often ignore essential practices that help to improve the health status of individuals [17].

In this study, schools are avenues of social influence and potentially harmful health practices as expressed by parents. Children and adolescents preferred school food; however, parents and health teams complained about the quality of school food, which in Saudi Arabia is reported to be high in carbohydrate. 
Within schools, it is essential to implement policies that promote health through adopting healthy life style and disseminating health messages [18]. Culture was also found to have an effect in this study, two fathers expressed their levels of knowledge and involvement in their children's care differed. One of them was involved in his child's treatment while the other father depended on his wife and gave her complete responsibility. That seems to echo Alsaleh study in the Saudi culture's stereotyping of the father's exclusive role as a breadwinner, which still plays a role in fathers' involvement [19]. Moreover, the majority of children and adolescents in this study were active and practised their favourite exercise. However, three young females preferred a sedentary lifestyle because of the difficulties of exercising due to the hot weather or the influence of cultural norms. In late 2017, regulations for female physical activity in school were introduced, and the Saudi government applied a tax on energy and soft drinks; thus, this might limit the future consumption of these drinks. This regulation was applied after the data collection.

\section{Limitation of the Study}

This study was conducted in two children diabetic clinic at two hospitals in Saudi Arabia. The target sample was children and adolescents with Type 1 diabetes and their parents. The sample size 32 was small and may not represent all the children in Saudi Arabia. Results cannot be generalized. However, this study findings might be used theoretically to national study on other children with Type1 diabetes in a similar context.

\section{Conclusion}

The study findings indicate a requirement to employ innovative and engaging ways of communicating with children and adolescents concerning the promotion of a positive lifestyle and healthy practices. In most cases, young people are faced with many challenges that affect their health, and limited care is taken to ensure that they are safe from them. There is a need for health professionals to reassess the children's and parents' knowledge of Type 1 diabetes hypoglycaemia management at every opportunity. Parents need back-up support and information to help them to recall what they need to do, such as colourful, simply worded information pamphlets. Moreover, there is a need to involve fathers in the care, because studies show the importance of fathers' involvement in their children's care. Fathers' involvement was seen to be facilitating their children's adherence to Type 1 diabetes management. There is a need for cooperation between schools and health ministries to increase awareness about Type 1 diabetes management and complications and promotion of healthy lifestyle.

\section{Conflicts of Interest}

The authors declare no conflicts of interest regarding the publication of the paper. 


\section{References}

[1] International Diabetes Federation (2017) IDF Diabetes Atlas. 8th Edition, International Diabetes Federation, Brussels. http://www.diabetesatlas.org

[2] American Diabetes Association (2018) Statistics about Diabetes. http://www.diabetes.org/diabetes-basics/statistics

[3] Al-Rubeaan, K. (2015) National Surveillance for Type 1, Type 2 Diabetes and Prediabetes among Children and Adolescents: A Population-Based Study (SAUDI-DM). Journal of Epidemiology and Community Health, 69, 1045-1051. https://doi.org/10.1136/jech-2015-205710

[4] Gomes, M.B., Santos, D.C., Pizarro, M.H., Barros, B., de Melo, L. and Negrato, C.A. (2018) Does Knowledge on Diabetes Management Influence Glycemic Control? A Nationwide Study in Patients with Type 1 Diabetes in Brazil. Patient Preference and Adherence, 12, 53-62. https://doi.org/10.2147/PPA.S146268

[5] Moawad, S., Badawy, A.S., Al-Saffar, Z.A., Al-Hamdan, N. and Awadien, A.M. (2014) Assessment of Knowledge among Saudi Diabetic Children/Adolescent at Riyadh City. American Journal of Nursing, 3, 5-12. https://doi.org/10.11648/j.ajns.20140301.12

[6] Beshi, S., Moawad, S. and Yakout, S. (2017) Assessment of Knowledge of Saudi Female Teenagers \& Their Mothers Regarding Management of Type I Diabetes in the City of Abha. Journal of Nursing Education and Practice, 7, 121. https://doi.org/10.5430/jnep.v7n9p121

[7] Pelicand, J. (2015) Self-Care Support in Paediatric Patients with Type 1 Diabetes: Bridging the Gap between Patient Education and Health Promotion? A Review. Health Expectations, 18, 303-311. https://doi.org/10.1111/hex.12041

[8] Bibace, R. and Walsh, M.E. (1980) Development of Children's Concepts of Illness. Pediatrics, 66, 912-917.

[9] Piaget, J. (1976) Piaget's Theory. In: Inhelder, B., Chipman, H.H. and Zwingmann, C., Eds., Piaget and His School, Springer Study Edition, Springer, Berlin, Heidelberg, 11-23. https://doi.org/10.1007/978-3-642-46323-5 2

[10] Grylli, V., Hafferl-Gattermayer, A., Wagner, G., Schober, E. and Karwautz, A. (2005) Eating Disorders and Eating Problems among Adolescents with Type 1 Diabetes: Exploring Relationships with Temperament and Character. Journal of Pediatric Psychology, 30, 197-206. https://doi.org/10.1093/jpepsy/jsi007

[11] Eiser, C. (1990) Psychological Effects of Chronic Disease on Children and Their Families. The Journal of Psychology and Psychiatry, 31, 85-98. https://doi.org/10.1111/j.1469-7610.1990.tb02274.x

[12] Archinkova, M., Konstantinova, M., Savova, R., Iotova, V., Petrova, C., Kaleva, N., Mladenov, W., et al. (2018) Glycaemic Control among Bulgarian Children and Adolescents with Type 1 Diabetes-An Impact of the Social Status and the Educational Level of the Parents. Biotechnology \& Biotechnological Equipment, 32, 535-541. https://doi.org/10.1080/13102818.2018.1429309

[13] Haugstvedt, A., Wentzel-Larsen, T., Rokne, B. and Graue, M. (2011) Perceived Family Burden and Emotional Distress: Similarities and Differences between Mothers and Fathers of Children with Type 1 Diabetes in a Population-Based Study (Clinical Report). Pediatric Diabetes, 12, 107-114. https://doi.org/10.1111/j.1399-5448.2010.00661.x

[14] Stefanowicz, A., Mysliwiec, M. and Adamkiewicz-Drozynska, E. (2018) Parental Knowledge and Metabolic Control of Children and Young Adults with Type 1 Dia- 
betes. Archives of Medical Science, 14, 52-59.

https://doi.org/10.5114/aoms.2015.53832

[15] Opondo, C. (2016) Father Involvement in Early Child-Rearing and Behavioural Outcomes in Their Pre-Adolescent Children: Evidence from the ALSPAC UK Birth Cohort. BMJ Open, 6, 10 p. https://doi.org/10.1136/bmjopen-2016-012034

[16] Shen, B., Centeio, E., Garn, A., Martin, J., Kulik, N., Somers, C. and Mccaughtry, N. (2018) Parental Social Support, Perceived Competence and Enjoyment in School Physical Activity. Journal of Sport and Health Science, 7, 346-352.

https://doi.org/10.1016/j.jshs.2016.01.003

[17] Hills, A., Dengel, D. and Lubans, D. (2015) Supporting Public Health Priorities: Recommendations for Physical Education and Physical Activity Promotion in Schools. Progress in Cardiovascular Diseases, 57, 368-374.

https://doi.org/10.1016/j.pcad.2014.09.010

[18] Ickovics, J., Duffany, K., Shebl, F., Peters, S., Read, M., Gilstad-Hayden, K. and Schwartz, M. (2019) Implementing School-Based Policies to Prevent Obesity: Cluster Randomized Trial. American Journal of Preventive Medicine, 56, e1-e11. https://doi.org/10.1016/j.amepre.2018.08.026

[19] Alsaleh, S.A. (2012) Gender Inequality in Saudi Arabia: Myth and Reality. International Proceedings of Economics Development \& Research, King Saud bin Abdulaziz University for Health Sciences Riyadh, 39, 12. 